\title{
I. INTRODUCTION TO THE PRACTICAL MANUAL
}

This practical manual is designed to complement the theoretical manual and to provide the trainer with simple practical tasks that reinforce and enhance comprehension of theoretical training on horticultural chain management. The trainer may in turn use this guide for the development of context-appropriate hands-on training packages for small-scale farmerlearner programmes.

The manual includes demonstrations, hands-on activities, tasks (such as the development of questionnaires and the conduct of interviews), problem-solving challenges and field visits with a focus on observation and recording. The following approaches have been selected for inclusion in this manual:

- Experiments with demonstrations: These include introductory presentations followed by practical demonstrations and a hands-on 'do-it-yourself' exercise. Each individual must take notes, make daily observations and record results. A report, which includes data analysis, discussions and conclusions, is required at the end of the training programme with verbal feedback of results and a group discussion on the effectiveness of the practical exercise. Practical exercises are designed to be very basic and inexpensive, with minimal requirements for equipment and/or chemicals, such that they can be easily duplicated under field conditions.

- Tasks: Tasks have been selected to provide the participant with a basic understanding of the issues at stake. The discussion sessions emanating from each task provide an opportunity for participants to contribute their experiences and to contextualise the theoretical work.

- Problem solving: This approach refers to brief discussions of classic case studies, which allows trainees to apply lesson concepts as they work through each problem.

- Field visits: The guide to field visits provides some basic background information on the site to be visited and highlights key points that should be observed during the site visit. 